\title{
Lysozyme interaction with negatively charged lipid bilayers: protein aggregation and membrane fusion
}

\author{
Tamer Al Kayal, ${ }^{a}$ Silvia Nappini, ${ }^{a}$ Edda Russo, ${ }^{b}$ Debora Berti, ${ }^{a}$ Monica Bucciantini,,${ }^{b c}$ Massimo Stefani ${ }^{* b c}$ \\ and Piero Baglioni $* a$
}

\author{
Received 6th October 2011, Accepted 26th January 2012 \\ DOI: $10.1039 / \mathrm{c} 2 \mathrm{sm} 06906 \mathrm{~g}$
}

\begin{abstract}
We report on the interaction of hen egg-white lysozyme (HEWL) with lipid vesicles in terms of surfaceinduced protein conformational variation and subsequent aggregation. In particular, we investigated the variations of the secondary structure of native lysozyme in the presence of liposomes with different surface charge density, resulting from different molar ratios of the zwitterionic POPC (1-palmitoyl-2oleoyl-sn-glycero-3-phosphocholine) and the negatively charged POPG (1-palmitoyl-2-oleoyl-snglycero-3-phospho-(1'-rac-glycerol)). It is well known that the main driving force involved in the interaction between globally anionic liposomes and lysozyme is electrostatic compensation, which, in some cases, produces extended aggregation. Moreover the presence of membranes can induce unfolding in the protein. In order to understand the main determinants of such phenomena, we probed simultaneously lysozyme-induced vesicle fusion events, variations in the secondary structure of the protein and its effect on liposomal membrane fluidity. We found that above a charge-density threshold, the association with vesicles results in modifications of the native structure associated with a decrease of liposomal membrane fluidity. Electron microscopy images revealed that the above described interactions result in mesoscopic structural changes, i.e. liposome clustering and fusion, together with the appearance of elongated structures, reminiscent of fibrillar aggregates. Additionally, a confocal microscopy analysis revealed that upon interaction with giant unilamellar vesicles (GUVs) of the same lipid composition where the above interactions were observed, a prompt insertion of lysozyme in the membrane occurs, leading to vesicle clustering, with the appearance of elongated structures where both the lipid and the protein are present.
\end{abstract}

\section{Introduction}

Protein fibrillization plays a critical role in the pathogenesis of a number of diseases involving the deposition in various organs and tissues of aggregates of a number of different proteins or peptides. ${ }^{1}$ The bulk of these deposits is composed of amyloid fibrils, filamentous polymeric structures displaying an ordered $\beta$ sheet-rich core where the $\beta$-strands of the constituting polypeptide chains run perpendicular to the major fibril axis, forming a couple of parallel $\beta$-sheets running along it (the so-called cross$\beta$ structure). ${ }^{2}$

Hen egg-white lysozyme (HEWL) is a small globular, strongly basic protein $(\mathrm{pI}=11.0)$ bearing a net positive charge over

${ }^{a}$ Department of Chemistry, University of Florence and CSGI, Via della Lastruccia 3-Sesto Fiorentino, 50019 Florence, Italy.E-mail: baglioni@, csgi.unifi.it

${ }^{b}$ Department of Biochemistry, University of Florence, Viale Morgagni, 50, 50134 Florence, Italy

'Research Centre on the Molecular Basis of Neurodegeneration (CIMN), University of Florence, Viale Morgagni, 50, 50134 Florence, Italy. E-mail: Massimo.stefani@unifi.it a broad $\mathrm{pH}$ range with a high affinity for anionic phospholipid bilayers, ${ }^{3}$ whose structure, activity and physicochemical properties have been thoroughly studied. ${ }^{4-6}$ HEWL is structurally homologous to human lysozyme, involved in several destabilizing mutations - related to protein deposition into amyloid plaques found in the liver, kidneys, and spleen of patients affected by non-neuropathic hereditary amyloidosis. ${ }^{7-11}$ Because of its homology to human lysozyme, HEWL has been extensively used as a model in studies for a number of investigations: protein adsorption at interfaces, ${ }^{12,13}$ membrane fusion, ${ }^{14-17}$ protein folding and aggregation into amyloids. ${ }^{18-21}$ Human lysozyme forms amyloid fibrils in vitro upon incubation at low $\mathrm{pH}$ and elevated temperature, ${ }^{22,23}$ whereas HEWL forms amyloid fibrils when incubated in organic solvents ${ }^{24}$ or in the presence of negatively charged lipid membranes. ${ }^{20}$

Lysozyme strongly binds to negatively charged membranes via electrostatic interactions, resulting in loosening and destabilisation of the whole protein conformation, and in the alteration both of the membrane surface and of the bilayer core structure. $^{25,26}$ In spite of the key importance of the electrostatic interactions, an important role in promoting lysozyme binding to 
the lipid bilayer is played by hydrophobic interactions too. ${ }^{27}$ Previous studies have addressed the effect of lysozyme charge modulation, by variation of both $\mathrm{pH}$ and ionic strength, on its interaction with negatively charged phospholipid vesicles, and on the physicochemical properties of both the bound protein and the lipid membrane regions involved in such interactions, resulting in membrane destabilization. ${ }^{28,29}$

Membrane association with lysozyme is a multistep process thought to involve both electrostatic and hydrophobic interactions. ${ }^{10,17,23,26,27}$ The steps include: (1) the initial protein adsorption on the lipid bilayer surface driven by ionic and/or hydrogen bond-mediated contacts; (2) the conformational modification of the protein; (3) the structural reorganization of the lipid phase; and (4) the partial insertion of the protein into the hydrophobic core of the bilayer. In agreement with a generally recognized concept, it has been suggested that the structural transition of lysozyme to a partially unfolded, aggregation-prone state is a critical prerequisite for fibrillization. ${ }^{30}$ However, in spite of the considerable progress achieved in understanding the mechanisms and pathways of lysozyme fibrillization, the molecular details and consequences of its interaction with lipid membranes remain poorly understood. ${ }^{31}$

Protein-mediated cell fusion is a widespread process in cell biology occurring in exocytosis, intracellular vesicle trafficking and others. ${ }^{32}$ However, there is a substantial lack of knowledge on the molecular and physicochemical basis of protein interaction with artificial membranes as well as of protein-mediated interaction of the two opposing outer lipid leaflets. ${ }^{33,34}$ Recently, lysozyme has been reported to induce $\mathrm{pH}$-dependent fusion of phosphatidylserine vesicles below $\mathrm{pH} 5.0$ with protein penetration inside the lipid bilayer; ${ }^{35}$ however, other authors have reported that lysozyme shows fusion efficiency over a broad $\mathrm{pH}$ range with extensive mixing of phospholipids, but not vesicle content, suggesting vesicle aggregation without effective fusion. $^{36}$

The present investigation was aimed at improving the knowledge of the effects of different HEWL concentrations adsorbed onto lipid vesicles containing various phospholipids, mixed in different ratios to vary the density of the surface negative charge. In particular our purpose was to gain a deeper understanding on the structural changes that occur simultaneously in the protein and the vesicle membrane upon interaction. These changes lead, in turn, to aggregation and variation of the fusogenic and stability properties of the HEWL/liposome system. Zwitterionic 1-palmitoyl-2-oleoyl-sn-glycero-3-phosphocholine (POPC) lipid vesicles and mixtures of POPC with the negatively charged 1-palmitoyl-2oleoyl-sn-glycero-3-phospho-(1'-rac-glycerol) (POPG) vesicles were used as model membranes with different surface charge densities. We investigated the effects of HEWL on the membrane fluidity of liposomes from POPC/POPG by measuring the variation of 1,6-diphenyl-1,3,5-hexatriene (DPH) fluorescence polarization. The effects of phospholipid vesicles on the secondary structure of the protein and on protein aggregates were investigated by means of Circular Dichroism (CD) and Thioflavin $\mathrm{T}$ (ThT) binding assay. Finally, a confirmation of the previous observations and a direct visualization of the aggregation phenomena were imaged by Transmission Electron Microscopy (TEM) and Confocal Laser Scanning Microscopy (CLSM).
CLSM is the suitable complementary technique for the above outlined experimental methods, as it permits a direct visualization of the interaction between giant unilamellar vesicles (GUVs) and HEWL. In fact, the choice of fluorescent tags with different emission wavelengths allowed distinguishing protein from lipids. Therefore, labeling GUVs with the lipid probe 1-oleoyl-2-\{6-[(7-nitro-2-1,3benzoxadiazol-4-yl)amino]hexanoyl\}-sn-glycero-3-phosphocholine (POPC-NBD) and labeling HEWL with 5-carboxytetra methylrhodamine (5-TAMRA) allowed direct observation of the protein fusogenic activity. Moreover, thanks to the selective staining of lipids and protein, a visualization of the formed structures was possible, as well as a 3D image acquisition of these aggregates by CLSM.

Our results can also be relevant to clarify the mechanism of the bactericidal and tumoricidal action of lysozyme and other amyloidogenic proteins. In fact, previous research has stressed that lysozyme cytotoxicity is related to its ability to penetrate into the lipid bilayer and to permeabilize the cell membrane upon aggregation. ${ }^{20,37,38}$

\section{Results and discussion}

\section{HEWL effects on liposomes: aggregation and alteration of the fluidity of the phospholipid membrane}

The effect of different HEWL concentrations on the size of the aggregates grown in the presence of POPG liposomes was investigated by Dynamic Light Scattering (DLS). The samples were mixed and incubated at room temperature for $20 \mathrm{~min}$ before measurements. The autocorrelation functions were inverted with the CONTIN algorithm to minimize possible artefacts of the Laplace inversion. The results are shown in Fig. 1 as intensityweighted size distributions. In the absence of HEWL, the vesicles displayed a narrow size distribution centred around $\sim 65 \mathrm{~nm}$ diameter. After incubation with $0.05 \mathrm{mg} \mathrm{ml}^{-1} \mathrm{HEWL}$ two new populations of scatterers, with diameters of $\sim 250$ and $\sim 1000 \mathrm{~nm}$, respectively, appeared, indicating vesicle aggregation/fusion,

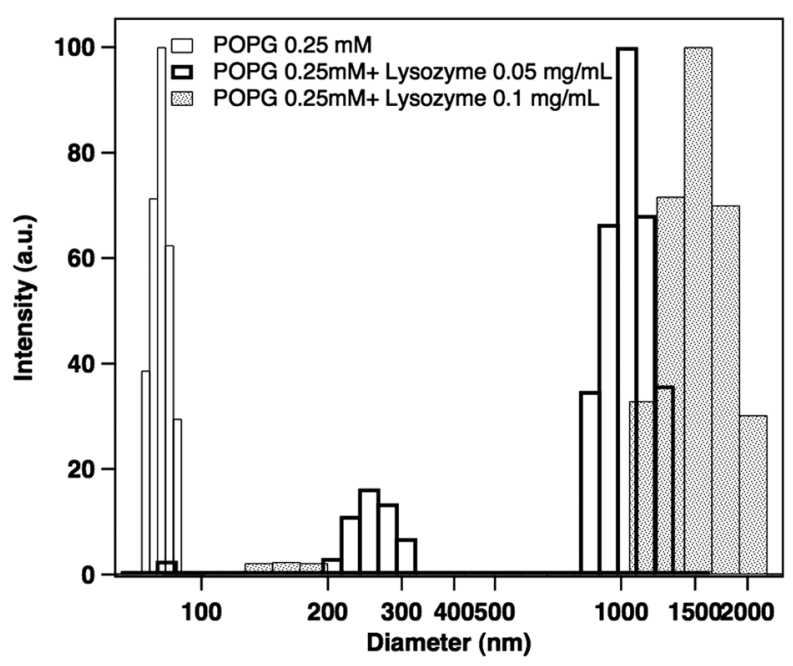

Fig. 1 Size distributions of the POPG liposome/HEWL system at different protein concentrations: $0.25 \mathrm{mM}$ POPG liposomes $\square$ without HEWL, $\boldsymbol{\square}$ with $0.05 \mathrm{mg} \mathrm{ml}^{-1}$ of HEWL, and $\square$ with $0.1 \mathrm{mg} \mathrm{ml}^{-1}$ of HEWL. 
together with an apparently modest residual distribution of the original vesicle population. Incubation with $0.1 \mathrm{mg} \mathrm{ml}^{-1} \mathrm{HEWL}$, corresponding to a $\sim 1056 \mathrm{HEWL} /$ liposome molar ratio, resulted in protein-vesicle complexes with two size distributions with diameters of $\sim 180$ and $\sim 1500 \mathrm{~nm}$, respectively, and the complete disappearance of the initial unperturbed liposome population, indicating that all the vesicles were involved in aggregation.

These data support an aggregation and/or fusogenic activity of HEWL on pure POPG vesicles favoured by the high density of negative charge, in agreement with previous results. ${ }^{36}$

Interestingly, the size distribution is, for these samples, bimodal, and characterized by the presence of a cluster of several liposomes, and a population characterized by micron-sized aggregates. A similar fusogenic activity leading to vesicle aggregation has been reported for other proteins and peptides. ${ }^{38-40}$

The ability of HEWL to perturb lipid bilayers, as a function of the negative charge density, was investigated by measuring the membrane fluidity through the fluorescence polarization $(P)$ of 1,6-diphenyl-1,3,5-hexatriene (DPH). DPH is a fluorescent probe that distributes throughout the hydrocarbon region of the membrane bilayer, whose fluorescence polarization $(P)$ is sensitive to variations of membrane fluidity. ${ }^{41}$

As Fig. 2 shows, we observed no changes of membrane fluidity in vesicles composed of pure POPC incubated with the whole range of HEWL concentrations, indicating that, under these experimental conditions, the protein does not apparently penetrate inside the bilayer. Conversely, an increase of HEWL concentration was associated to a decrease of membrane fluidity for vesicles containing $>10 \%$ POPG. Moreover, in the presence of such detectable protein-vesicle interactions, a saturation threshold was evident around $0.3 \mathrm{mg} \mathrm{ml}^{-1} \mathrm{HEWL}$, corresponding to a protein/liposome molar ratio of $\sim 3162$, i.e. well below the liposomal saturation coverage, which is around $10^{5} \mathrm{HEWL}$ molecules/liposome, considering a cross section of $13.5 \AA^{2}$ for the protein. This phenomenon is a consequence of the restriction of

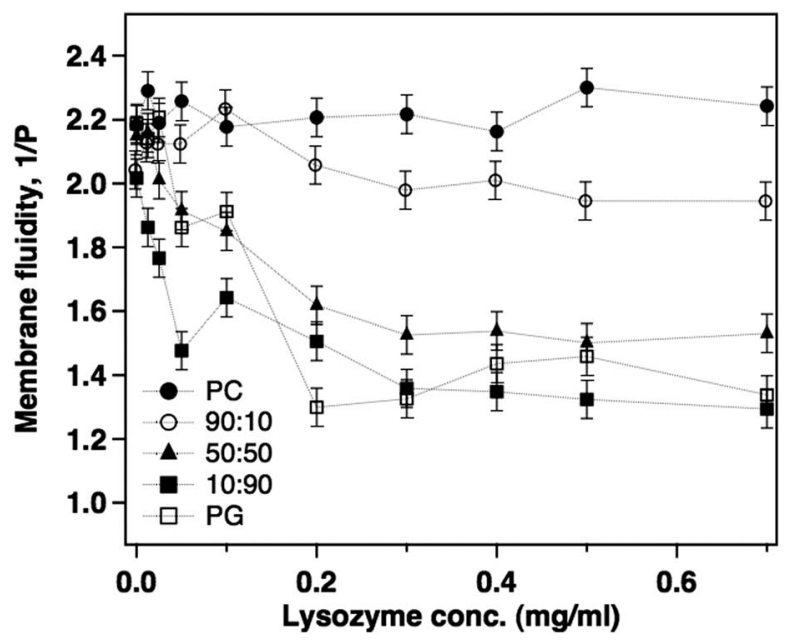

Fig. 2 Effect of HEWL concentration on the membrane fluidity of 0.25 $\mathrm{mM}$ liposomes prepared at different POPC/POPG molar ratios. Pure POPC -; POPC/POPG $90: 10$ O; POPC/POPG $50: 50 \boldsymbol{\Delta}$; POPC/ POPG $10: 90 \mathbf{m}$; pure POPG $\square$. The graph reports the standard deviation of three different measures for each system. the DPH rotational mobility due to a decreased rate of transgauche isomerization of hydrocarbon chains.

The negatively charged lipid membrane attracts cationic protein (lysozyme) to its surface, where it finds an anisotropic environment. The dynamic nature of the lipid bilayer, characterized by conformational fluctuations, allows a mutual adjustment of both lipid and protein conformations and localization to occur. ${ }^{17}$ Immediately after its interaction within the lipid bilayer, lysozyme undergoes conformational changes with subsequent reorganization (oligomerization) that leads to vesicle clustering and amyloid fibril formation. ${ }^{42-45}$ The concomitant presence of these effects contributes to a decrement of the lipid membrane fluidity $(1 / P)$.

These data agree with previous reports on $\mathrm{A} \beta 40$ and $\operatorname{PrP}^{46,47}$ and indicate the importance of the lysozyme/liposome ratio in modulating not only vesicle aggregation, but also membrane fluidity, most likely resulting from protein insertion within the bilayer, as shown for many other proteins. ${ }^{48}$ However, the reported alteration of membrane fluidity in the presence of HEWL can also be the result of other modifications of the bilayer such as aggregate-induced lipid dehydration, ${ }^{49}$ reduction in bilayer free volume, presumably caused by an increased packing density of hydrocarbon chains ${ }^{50}$ or any alteration of the order of the phospholipid tails.

Such results suggest that both the occurrence and the extent of HEWL interaction with the lipid bilayer of liposomes depend on protein concentration and charge density at the vesicle surface. Therefore the protein, recruited by electrostatic driving forces at the membrane surface, both reduces the membrane fluidity through the partial insertion into the lipid core and screens the electrostatic repulsion between the negatively charged liposomes.

These results are also in agreement with previous findings showing local demixing in phospholipid vesicles incubated in the presence of lysozyme. $^{51}$

\section{Structural modifications of HEWL upon interaction with negatively charged vesicles}

Once the effect of HEWL on the stability and fluidity of vesicle bilayers at different negative charge densities at the surface was established, we focused our attention on the effects of vesicles with different POPC : POPG molar ratios on the HEWL structure and aggregation, by Circular Dichroism (CD) analysis and Thioflavin $\mathrm{T}$ (ThT) assay. CD analyses were carried out on vesicle-protein mixtures incubated at room temperature for different time intervals (20 min, 2 days and 30 days). A modification of the HEWL native structure upon incubation with $100 \%$ POPG and 10:90 POPC : POPG vesicles was clearly observed. Upon incubation, HEWL showed progressive modifications of the secondary structure (Fig. 3a and b). In particular, the CD spectrum obtained incubating $0.1 \mathrm{mg} \mathrm{ml}^{-1} \mathrm{HEWL}$ with $0.25 \mathrm{mM}$ pure POPG vesicles (Fig. 3a) for 20 min indicated a transition from the native secondary structure to one with increased content of $\beta$-sheet suggesting that the protein underwent structural modifications at the vesicle surface. After $48 \mathrm{~h}$ of incubation, a CD spectrum typical of the presence of $\alpha$-structure was seen, and this structure persisted unchanged until one month of incubation, indicating the stabilization of this kind of secondary motif for the protein. However $0.1 \mathrm{mg} \mathrm{ml}^{-1} \mathrm{HEWL}$ behaved 

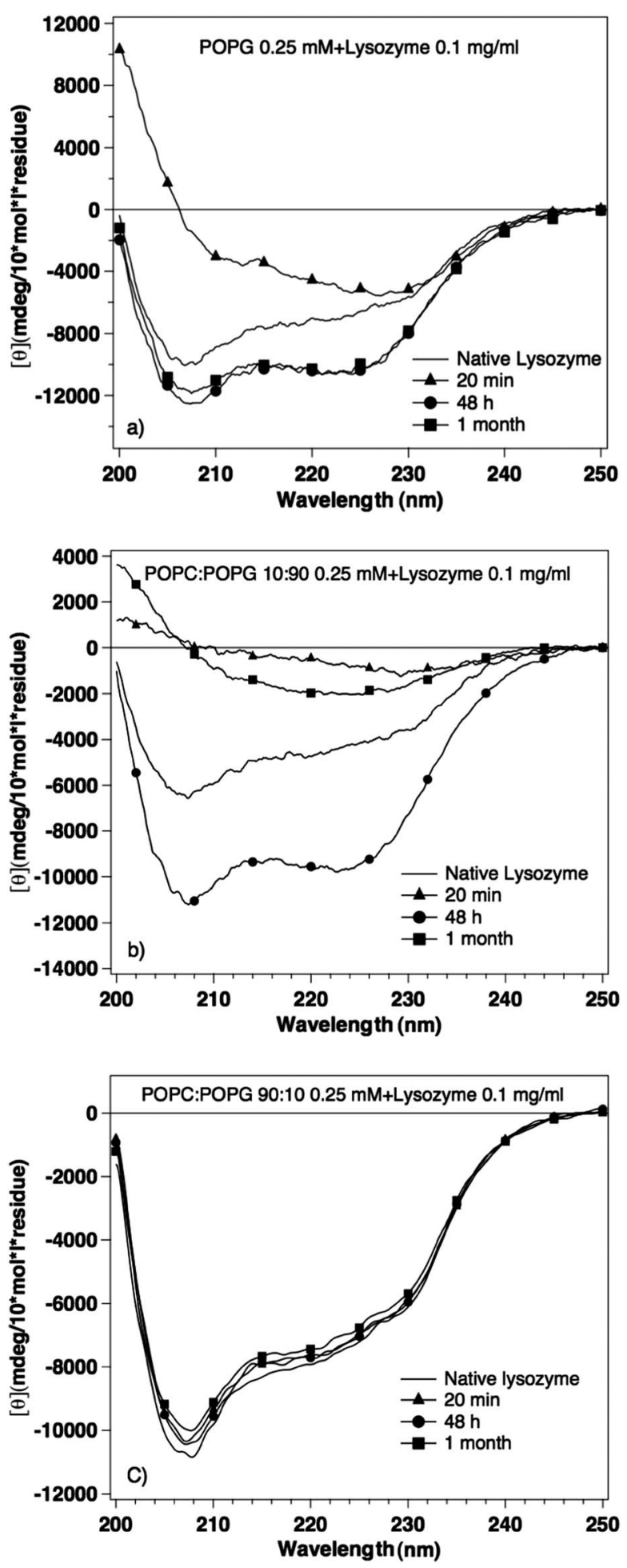

Fig. $3 \mathrm{CD}$ spectra of $0.1 \mathrm{mg} \mathrm{ml}^{-1} \mathrm{HEWL}$ in $10 \mathrm{mM}$ sodium phosphate buffer, $\mathrm{pH}$ 7.3, measured as a function of time after the addition of $0.25 \mathrm{mM}$ of pure POPG (a), $10: 90$ POPC/POPG (b) or $90: 10 \mathrm{POPC}$ POPG (c) vesicles.

differently when incubated in the presence of $10: 90 \mathrm{POPC} /$ POPG vesicles. In fact, in this case, the CD spectrum, initially indicative of $\beta$-structure, became characteristic of $\alpha$-structure after $48 \mathrm{~h}$ of incubation and turned back to $\beta$-structure after one month of incubation (Fig. 3b). No effects on the native secondary structure of the protein were observed when HEWL was incubated with pure POPC vesicles or with $90: 10 \mathrm{POPC} /$ POPG vesicles (Fig. 3c). Therefore the association of the protein with the bilayer, with possible insertion inside it, as monitored in terms of decrease of membrane fluidity, is accompanied by variation of the protein secondary structure. The type, extent and time evolution of such structural modifications is dependent on protein concentration.

These data are in good agreement with previous studies on $A \beta$, indicating different structural transitions of the peptide on the vesicle surface, where $\beta$-structure predominates favouring aggregation, or inside the bilayer, where $\alpha$-helix becomes stabilized favouring the persistence of the monomeric state. ${ }^{52}$ HEWL aggregation upon incubation for one month at neutral $\mathrm{pH}$ in the presence or in the absence of vesicles with different POPC : POPG ratios was also investigated with Thioflavin $T$ binding assays. Thioflavin $\mathrm{T}(\mathrm{ThT})$ is a fluorescent dye widely used to visualize and quantify the presence of amyloid aggregates. In fact, it binds selectively to $\beta$-sheet-rich structures, such as those found in amyloid aggregates, displaying enhanced fluorescence and a characteristic red shift of its emission spectrum. $0.5 \mathrm{mg} \mathrm{ml}^{-1}$ of HEWL was used in this assay to reduce the interference of large protein-vesicle aggregates with fluorescence readings. Under these conditions, HEWL did not aggregate in the absence of liposomes, whereas ThT fluorescence progressively grew in the presence of vesicles with increasing contents of POPG, suggesting the growth of ordered aggregates (Fig. 4).

These data substantiate previous results indicating that high densities of negative charge on liposome surfaces favour the formation of mixed protein-vesicle aggregates. ${ }^{53,54}$ In particular, we found that HEWL incubation with $90 \%$ POPG vesicles resulted in enhanced ThT fluorescence as compared to HEWL incubated with pure POPG vesicles. Such evidence agrees with the progression of the $\mathrm{CD}$ spectra (Fig. 3b) above reported and suggests that $90 \%$ POPG provides a density of negative charge that is optimal for fibril formation among the investigated

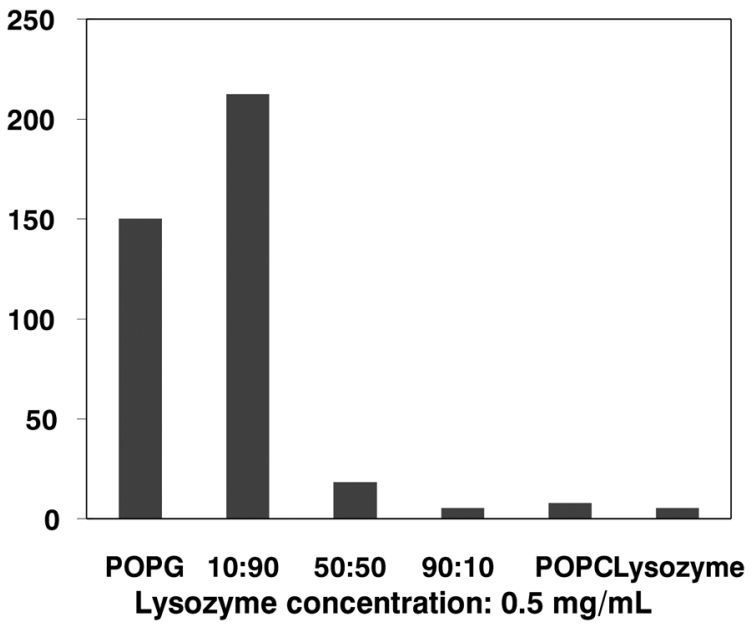

Fig. 4 Relative fluorescence of ThT monitored after lysozyme incubation with different molar ratios of POPG/POPC liposomes for one month. 
POPG/POPC ratios. This finding can tentatively be explained hypothesizing that pure POPG vesicles carry an excess of negative charge that disturbs the charge equilibrium between protein molecules and the vesicle surface; as a consequence, the HEWL structure could be differently affected possibly favouring its ability to insert inside the bilayer thus reducing its propensity to generate inter-molecular interactions among similarly misfolded protein molecules.

Alternatively, the effect could be explained in terms of a different number of HEWL molecules recruited at the vesicle surface with modifications of the spatial arrangement and of the ability to oligomerize the misfolded protein molecules. If so, the optimal density of negative charge is mirrored by a density of HEWL molecules at the vesicle surface that is the best for protein aggregation. Lateral density of the adsorbed protein in controlling the protein self-association behavior was previously proposed. ${ }^{55}$

The structural modifications of both vesicles and proteins in the HEWL-vesicle system were imaged by Transmission Electron Microscopy (TEM) and Confocal Laser Scanning Microscopy (CLSM) under the different experimental conditions used for the previous experiments.

TEM pictures of POPC/POPG vesicles incubated with 0.05 or $0.1 \mathrm{mg} \mathrm{ml}^{-1}$ of HEWL were acquired using the same $0.25 \mathrm{mM}$ phospholipid composition for CD experiments.
Pure POPG liposomes incubated with $0.05 \mathrm{mg} \mathrm{ml}^{-1} \mathrm{HEWL}$ for $48 \mathrm{~h}$ showed an initial vesicle aggregation (Fig. 5a), followed by an extended vesicle association and fusion after a prolonged incubation of 7 days (Fig. 5b).

TEM images of pure POPG vesicles incubated for 7 days with a higher HEWL concentration $\left(0.1 \mathrm{mg} \mathrm{ml}^{-1}\right)$ showed the formation of diffused filamentous structures, most likely composed of protein/lipid aggregates (Fig. 5c). Amyloid fibril-like structures coexisting with apparently amorphous materials were observed in 10:90 POPC/POPG liposome samples incubated for 7 days with $0.1 \mathrm{mg} \mathrm{ml}^{-1}$ HEWL (Fig. 5d).

Electron microscopy allowed high resolution imaging but not in situ observation; moreover, it did not provide a recognition of protein and phospholipids in the final aggregates. This experimental limitation was overcome by Confocal Laser Scanning Microscopy (CLSM), where the choice of different and spectroscopically complementary tags allowed distinguishing protein from lipids. Therefore, we further investigated the HEWL-lipid interaction by CLSM using giant unilamellar vesicles (GUVs) of pure POPC or POPG labeled with the lipid probe 1-oleoyl-2-\{6[(7-nitro-2-1,3-benzoxadiazol-4-yl)amino]hexanoyl\}-sn-glycero3-phosphocholine (POPC-NBD) and HEWL tagged with the 5-carboxytetramethylrhodamine (5-TAMRA) protein labeling kit.

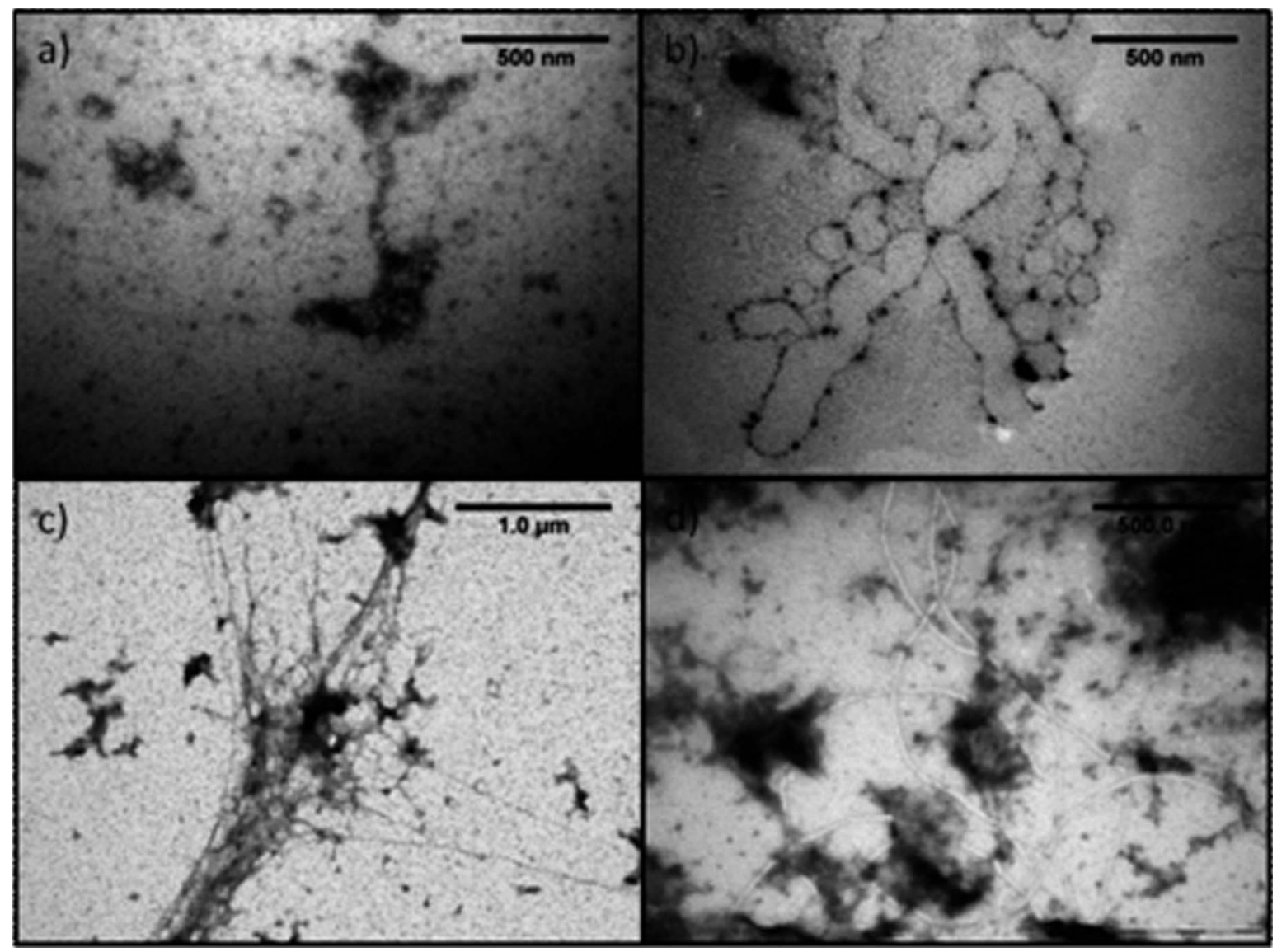

Fig. 5 TEM images of $0.25 \mathrm{mM}$ pure POPG vesicles incubated with $0.05 \mathrm{mg} \mathrm{ml}^{-1}$ HEWL for 2 days (a) and 7 days (b). (c) TEM images of $0.25 \mathrm{mM}$ POPG vesicles incubated with $0.1 \mathrm{mg} \mathrm{ml}^{-1}$ HEWL for 7 days. (d) TEM images of $0.25 \mathrm{mM} 10: 90$ POPC/POPG vesicles incubated with $0.1 \mathrm{mg} \mathrm{ml}^{-1}$ HEWL for 7 days. 
GUVs, with a diameter ranging between 1 and $100 \mu \mathrm{m}$, with identical local arrangement as liposomes, i.e. lipid bilayers, can be used as realistic, even if simplistic, models of cell membranes to visualize membrane perturbations with optical microscopic

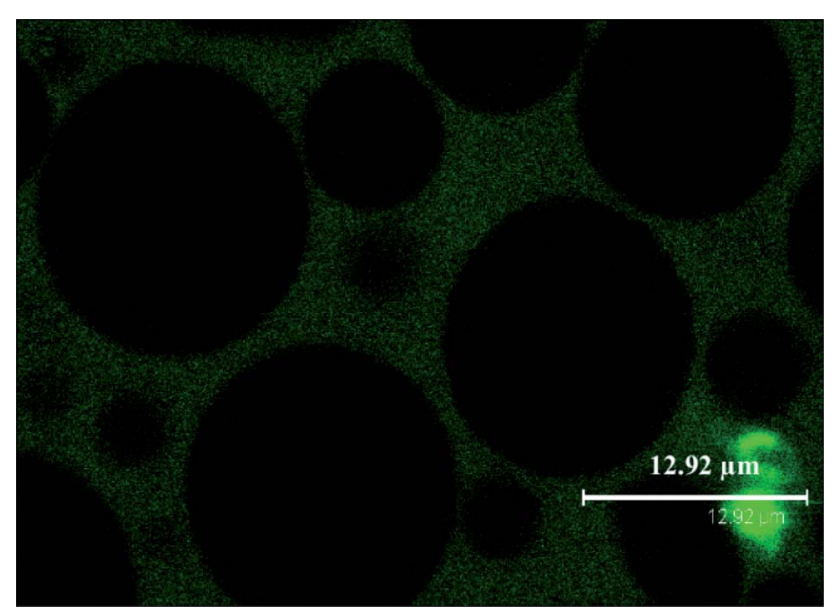

Fig. 6 CLSM imaging of POPC GUVs incubated with 5-TAMRAlabeled HEWL (green). techniques. ${ }^{56}$ In fact, GUV interaction with HEWL was easily visualized by CLSM directly in solution, providing interesting insights into the aggregation mechanism. No protein-vesicle interaction occurred when 5-TAMRA-labeled HEWL was added to a pure POPC GUV solution, in agreement with the results previously obtained with liposomes. Fig. 6 clearly shows that the labeled protein is confined outside the vesicles, yielding a uniform green background, with no sign of locally increased concentration at the membrane.

This observation agrees with the above reported lack of both HEWL-vesicle interaction and significant changes of membrane fluidity in pure POPC liposomes incubated with the protein.

Conversely, HEWL induced aggregation is clearly visible in a series of CLSM pictures taken at different times, for negatively charged pure POPG giant vesicles.

Fig. 7 shows a series of confocal images, obtained for fluorescent GUVs doped with $0.1 \% \mathrm{~mol} / \mathrm{mol} \mathrm{NBD}-P O P C$ (green) after the addition of 5-TAMRA-labeled HEWL (red). It is important to remark that colours for NBD-POPC and 5-TAMRA-labeled HEWL in the images were chosen arbitrarily by the CLSM operator, and are not intrinsic characteristics of the probes. The initial interaction of HEWL with GUV membranes, which appears mostly red-stained by 5-TAMRA-labeled HEWL

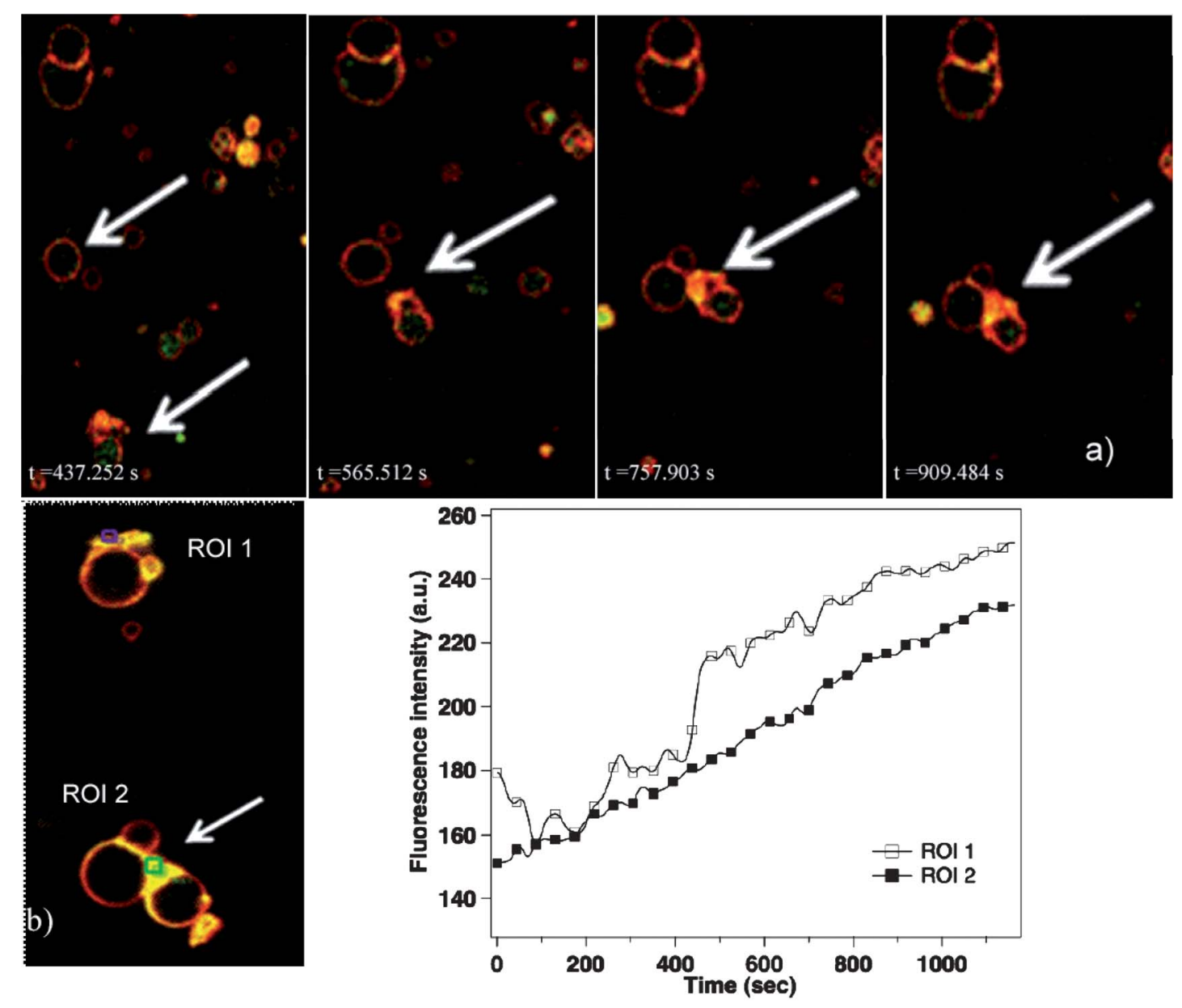

Fig. 7 (a) CLSM images taken at different times (acquisition mode xyt) of POPC-NBD-labeled GUVs (green) incubated with 5-TAMRA-labeled HEWL (red). (b) Fluorescence intensity of the POPC-NBD probe in different junction areas between GUVs (ROI 1: violet; ROI 2: green), monitored during the incubation period. 
adsorption, can be clearly visualized from these images. The interaction promptly induced vesicle clustering as indicated by the arrows. Moreover, NBD-POPC accumulation at the junction areas between vesicles was observed. We therefore monitored the increase of NBD-POPC fluorescence for about 20 min after GUV aggregation. The results indicated that HEWL interaction with GUVs induced phospholipid extraction from the membrane, as shown in Fig. 7b by the increase of NBD-POPC fluorescence in the selected junction points (ROI 1: violet and ROI 2: green). Phospholipid extraction supports HEWL insertion inside the bilayer under these conditions.

Similarly, CLSM images showed fusion and disruption of POPG GUVs labeled with POPC-NBD after the addition of a solution of unlabeled HEWL (Fig. 8). This behaviour is in

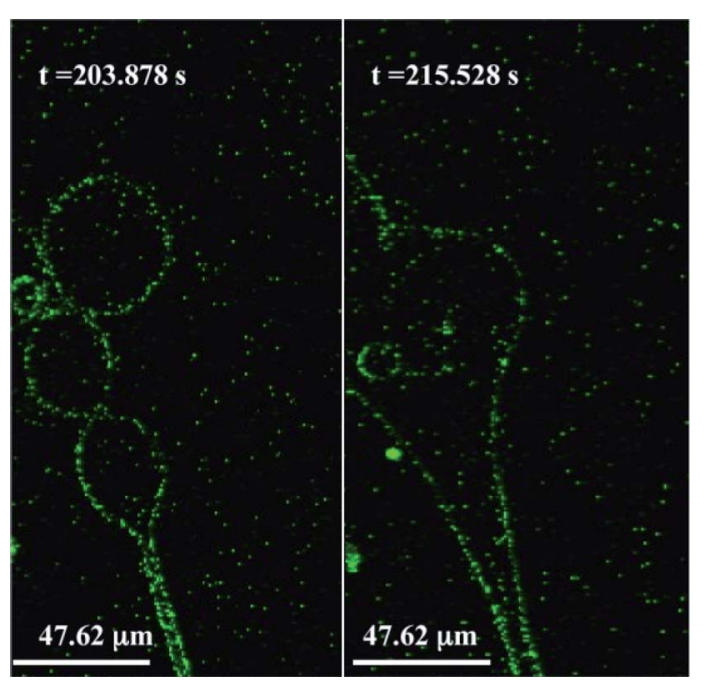

Fig. 8 CLSM images (acquisition mode xyt) showing the fusion of POPG GUVs $(0.1 \%$ mol POPC-NBD) incubated with unlabeled HEWL. agreement with the results observed for POPG liposomes incubated with HEWL and discussed in the TEM section (Fig. 5b).

Finally, we imaged the formation of extended filamentous structures of pure POPG GUVs labeled with $0.1 \%$ (molar ratio) NBD-POPC upon vesicle incubation with labeled HEWL for 7 days. Fig. 9 shows 3D confocal images of these structures showing that they are composed of both lipids (Fig. 9a) and proteins (Fig. 9b). Protein-lipid co-localization within the filamentous structures is shown in Fig. 9c, which clearly indicates that an interaction between them does exist. Finally, fibril growth was further confirmed by incubating for 7 days HEWL with unlabeled POPG GUVs in the presence of Thioflavin T (ThT).

Confocal microscopy images of HEWL/POPG GUVs treated with ThT showed the formation of fluorescent ThT-labeled filamentous structures indicative of the amyloid fibril formation (Fig. 10).

Fig. 11 shows a proposed schematic model of the events arising upon HEWL interaction with negatively charged vesicles. The positively charged protein is attracted by electrostatic forces at the vesicle surface, where it undergoes misfolding and subsequent aggregation, a step clearly influenced by $\mathrm{pH}$, ionic strength and lipid composition of the vesicles.

Depending on the density of charge at the vesicle surface, the protein can also penetrate, at least in part, inside the bilayer, where it is prevented from aggregating.

The presence of the surface-adsorbed protein molecules induces initially vesicle aggregation followed by lipid extraction, bilayer destabilization, vesicle fusion and subsequent disassembly.

\section{Materials and methods}

1-Palmitoyl-2-oleoyl-sn-glycero-3-phosphocholine (POPC), 1-palmitoyl-2-oleoyl-sn-glycero-3-phospho-(1'-rac-glycerol) (POPG) and the lipid probe 1-oleoyl-2-\{6-[(7-nitro-2-1,3-benzoxadiazol4-yl)amino]hexanoyl\}-sn-glycero-3-phosphocholine (NBD-PC)

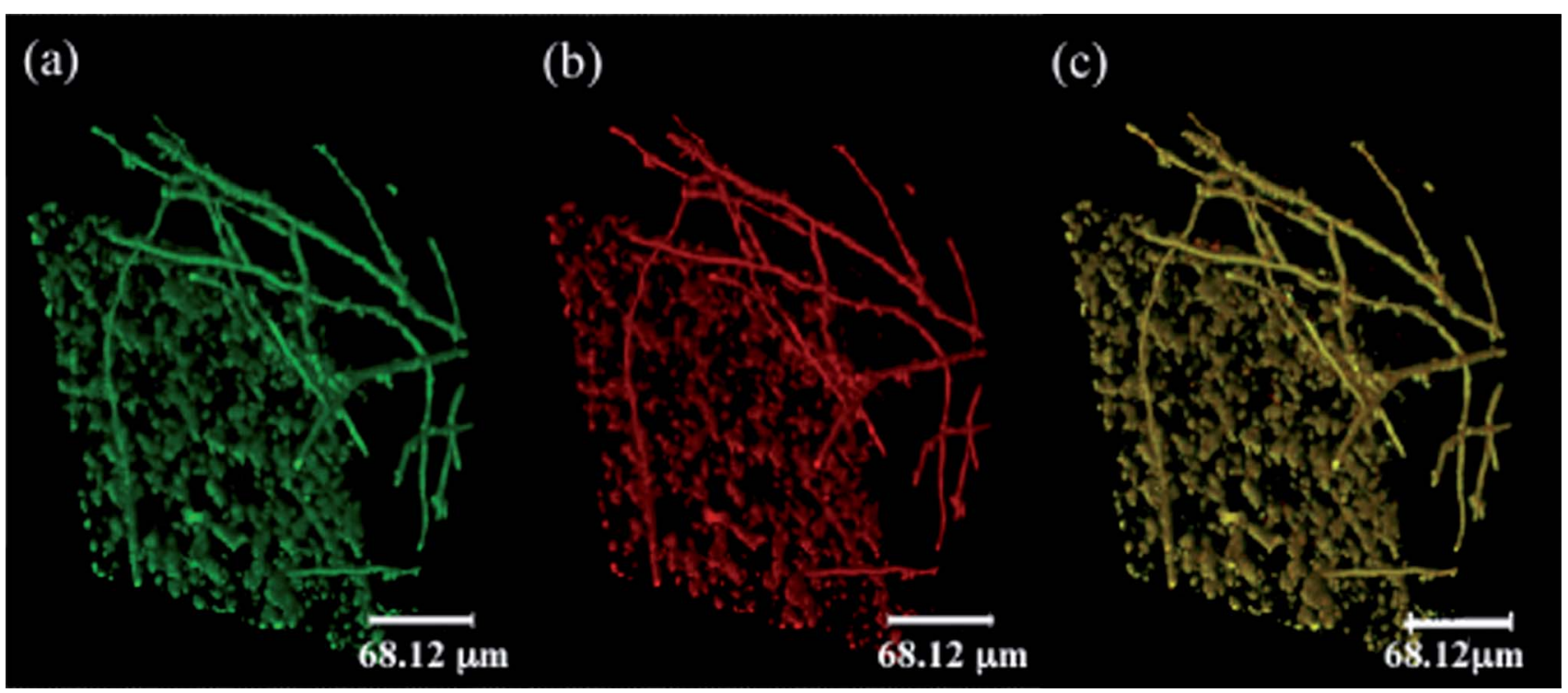

Fig. 9 Three-dimensional projection (max fix treatment) of POPG GUVs doped with $0.1 \%$ mol POPC-NBD incubated for 7 days with 5-TAMRAlabeled HEWL. (a) Image obtained by exciting the lipid marker (NBD-POPC), (b) image obtained by exciting the protein marker (5-TAMRA) and (c) overlay of the (a) and (b) images. 


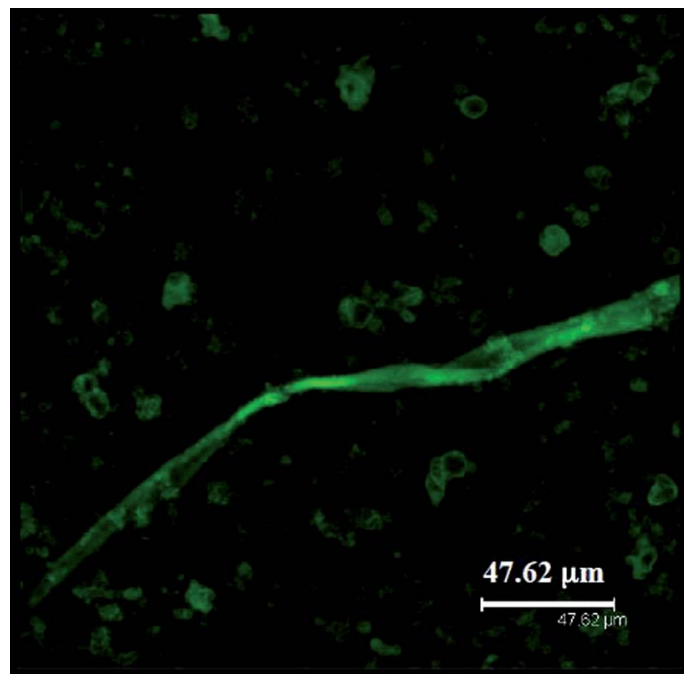

Fig. 10 Three-dimensional projection (max fix treatment) of unlabeled POPG GUVs incubated with HEWL for 7 days in the presence of Thioflavin $\mathrm{T}$ (green).

were purchased from Avanti Polar Lipids (Alabaster, AL). All reagents were of analytical grade or the highest purity available. 1,6-Diphenyl-1,3,5-hexatriene (DPH) was purchased from Molecular Probes (Invitrogen). AnaTagTM 5-TAMRA protein labeling kit was purchased from DBA Italia s.r.l. (Segrate, Italy).
Phosphate buffered saline (PBS), hen egg white lysozyme (HEWL), Thioflavin $\mathrm{T}$ (ThT) and other chemicals were purchased from Sigma (Milan, Italy) unless otherwise stated.

\section{Liposome preparation}

Lipid vesicles were prepared by the lipid film hydration method, followed by sequential extrusion. Appropriate amounts of concentrated lipid (POPC and POPG) stock solutions in chloroform were mixed and then diluted to $20 \mathrm{ml}$ with a chloroform/ methanol blend $(5: 1, \mathrm{v} / \mathrm{v})$. The organic solvent was removed using a rotatory evaporator.

The residual lipid film was kept under vacuum overnight, hydrated with buffer (10 mM sodium phosphate, $\mathrm{pH} 7.3)$ and vortex-mixed to produce multilamellar vesicles. The suspension was subjected to 10 freeze-thawing cycles to reduce multilamellarity and then repeatedly extruded through $100 \mathrm{~nm}(10$ times) and $50 \mathrm{~nm}$ (10 times) polycarbonate membranes using a Lipex Extruder (Northen Lipids Inc.). Size and size distribution of each liposomal preparation were controlled by DLS.

\section{Giant unilamellar vesicles}

GUVs were prepared using the electroformation method originally developed by Angelova and Dimitrov. ${ }^{57,58}$ A home-made chamber $^{56}$ was prepared by assembling two Indium Tin Oxide (ITO)-coated microscope slides separated by an O-ring spacer.

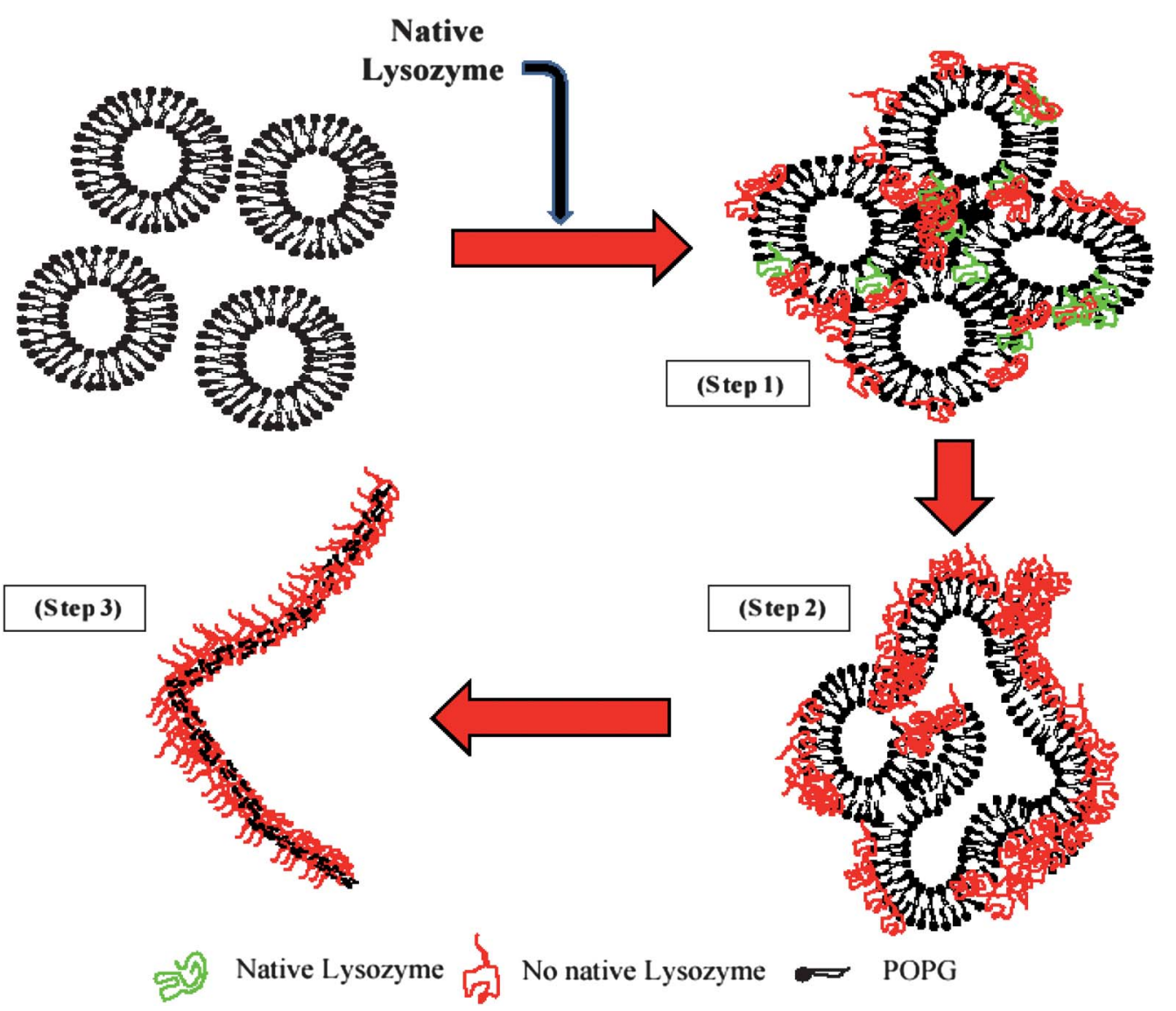

Fig. 11 A schematic model of the events arising upon HEWL interaction with negatively charged vesicles. 
The electrical connection with either side of the capacitor was obtained by directly attaching two $\mathrm{Cu}$ stickers on the conducting faces. The main reason for using ITO as an electrode is its transparency, so that the formation of giant vesicles could be easily observed using a microscope. The POPC and POPG solutions were dissolved in chloroform and a volume of $15 \mu \mathrm{l}$ was spread on each conducting face of ITO-coated microscope slides and then dried under vacuum for at least $2 \mathrm{~h}$ to remove the solvent. An O-ring was positioned around the film, and the two slides were sandwiched to form a chamber where GUVs growth took place. Then the chamber was filled with $350 \mu$ of a $0.25 \mathrm{M}$ sucrose solution and equilibrated at room temperature. Finally, the chamber was connected to a function generator and a lowfrequency AC electric field (sinusoidal wave with a frequency of $10 \mathrm{~Hz}$ and amplitude of $2 \mathrm{~V}$ ) was applied for $3 \mathrm{~h}$. When GUVs growth was complete, as monitored by an optical microscope, the solution was gently removed from the electro-formation chamber and the samples were diluted with an iso-osmolar $0.25 \mathrm{M}$ glucose solution. The density difference between the sucrose solutions inside and outside the vesicles leads to GUV deposition, favouring microscopic observation. Vesicles with 5$50 \mu \mathrm{m}$ diameter were obtained by the electro-formation method.

\section{Protein labelling}

HEWL was labeled with 5-TAMRA (5-carboxytetramethylrhodamine) by using the AnaTag 5-TAMRA protein labelling kit (Ana Spec). This kit provides a convenient way to label proteins by using the succinimidyl ester reactive form of 5TAMRA, which has a good reactivity and selectivity with the amino groups of the protein. The labeling process was performed following the standard protocol given by the provider. Further purification of the protein was done by dialysis in order to remove any excess of unreacted marker and to concentrate the HEWL solution by ultrafiltration.

\section{HEWL-liposome and HEWL-GUV mixtures}

HEWL was dissolved in $10 \mathrm{mM}$ sodium phosphate buffer, $\mathrm{pH}$ 7.3 , to a stock $2.0 \mathrm{mg} \mathrm{ml}^{-1}$ concentration. The liposome and lysozyme solutions were mixed to a $0.25 \mathrm{mM}$ final lipid concentration and varying final protein concentrations $(0.0125$, $0.025,0.050,0.1,0.2,0.3,0.4,0.5$ and $\left.0.7 \mathrm{mg} \mathrm{ml}^{-1}\right)$. In the case of GUVs, HEWL and 5-TAMRA-labeled HEWL were dissolved in $0.25 \mathrm{M}$ glucose. The lysozyme and GUV solutions were mixed to obtain a lysozyme/lipid molar ratio of $2.8 \times 10^{-5}$.

\section{Dynamic Light Scattering}

DLS experiments were carried out on an apparatus described elsewhere. ${ }^{59}$ The measurements were performed at $25{ }^{\circ} \mathrm{C}$ on $0.5 \mathrm{ml}$ samples previously transferred into disposable cells. For each sample the measurement was performed at the scattering angle $\theta=90^{\circ}$ corresponding to a scattering vector $\boldsymbol{q}=(4 \pi n / \lambda)$ $\sin (\theta / 2)$, where $n$ is the refractive index of the medium, $\lambda$ is the incident laser wavelength and $\theta$ is the angle between the incident light and the detector measuring the scattered intensity. In DLS experiments, the normalized time autocorrelation function of the scattered light electric field can be expressed as an intensity- weighted sum of exponential decays, each related to the Brownian motions of a given aggregate size.

$$
g_{1}(\boldsymbol{q}, \tau)=\int_{0}^{\infty} W(\Gamma) \exp (-\Gamma \tau) \mathrm{d} \Gamma
$$

The experimentally measured autocorrelation function is given by

$$
C(\boldsymbol{q}, \tau)=A\left(1+\beta^{2} g_{1}^{2}(\boldsymbol{q}, \tau)\right)
$$

where $A$ is the measured baseline, $\beta$ the spatial coherence factor, and $g_{1}(\boldsymbol{q}, t)$ the normalized electric field correlation function. For a dilute suspension of monodisperse particles, $g_{1}(\boldsymbol{q}, t)$ decays exponentially with a decay rate $\Gamma=D \boldsymbol{q}^{2}$, where $\boldsymbol{q}$ is the magnitude of the scattering wave vector, and $D$ is the translational diffusion coefficient, which is related to the hydrodynamic radius, $R_{\mathrm{H}}$, through the Debye-Stokes-Einstein relationship:

$$
R_{\mathrm{H}}=\frac{k_{\mathrm{B}} T}{6 \pi \eta D}
$$

where $\eta$ is the solvent viscosity and $k_{\mathrm{B}}$ the Boltzmann constant.

The analysis of the decay time distribution was carried out by the inverse Laplace transformation using the CONTIN method. ${ }^{60}$

\section{Membrane fluidity of liposome-HEWL complexes}

1,6-Diphenyl-1,3,5-hexatriene (DPH) fluorescence polarization was measured in the presence of HEWL and correlated with the membrane fluidity of the vesicles, as a function of vesicle composition. $^{21} \mathrm{DPH}$ is a neutral hydrophobic probe that distributes throughout the hydrocarbon region of the membrane bilayer. Any variation of membrane fluidity induces a modification of the DPH environment and hence of the DPH fluorescence polarization $(P)$. An appropriate volume of a $0.4 \mathrm{mM}$ DPH stock solution in ethanol was added to a preformed liposome suspension to obtain a lipid/DPH molar ratio $=250$. Then, the mixture was incubated for $1 \mathrm{~h}$ at room temperature before adding HEWL to obtain the different samples with $0.25 \mathrm{mM}$ final lipid concentration. The samples were incubated for $2 \mathrm{~h}$ before measurements.

The fluorescence polarization of the samples was measured by a LS50B spectrofluorimeter (Perkin Elmer, Italy). The samples were excited with vertically polarized light $(360 \mathrm{~nm})$, and emission intensities $(430 \mathrm{~nm})$ both parallel $\left(I_{\|}\right)$and perpendicular $\left(I_{\perp}\right)$ to the excited light were acquired. DPH polarization was calculated using the following equation:

$$
P=\frac{\left(I_{\|}-I_{\perp}\right)}{\left(I_{\|}+I_{\perp}\right)}
$$

Membrane fluidity of the liposome-HEWL complex was reported as reciprocal of polarization $(1 / P)$.

\section{Circular Dichroism}

CD spectra were recorded on a J-715 Jasco spectropolarimeter with a $50 \mathrm{~nm} \mathrm{m^{-1 }}$ scan speed; data points were collected from 250 to $190 \mathrm{~nm}$ at room temperature with a $0.1 \mathrm{~mm}$ Hellma quartz 
cylindrical cell and 2, $10 \mathrm{~mm}$ Hellma quartz cells. The observed CD was converted into molar ellipticity that was normalized for path length, amino acid residues and HEWL concentration.

\section{Thioflavin $\mathrm{T}$ assay}

$60 \mu \mathrm{L}$ of the different types of lipid suspensions were withdrawn at different incubation times and mixed with $440 \mu \mathrm{L}$ of $25 \mu \mathrm{M}$ ThT in $25 \mathrm{mM}$ phosphate buffer, pH 6.0. Sample fluorescence was measured at $25^{\circ} \mathrm{C}$ (440 $\mathrm{nm}$ excitation, $485 \mathrm{~nm}$ emission) in a $2 \times 10 \mathrm{~mm}$ path-length cell using a Perkin-Elmer LS 55 spectrofluorimeter (Wellesley, MA, USA) equipped with a thermostatted cell-holder connected to a Haake F8 water-bath (Karlsruhe, Germany).

\section{Electron and confocal microscopy}

Electron micrographs were acquired using a JEM 1010 transmission electron microscope at $80 \mathrm{kV}$ excitation voltage. After incubation, the lipid suspensions were adsorbed onto Formvar/ carbon-coated 400 mesh nickel grids (Agar Scientific, Stansted, UK) by floating the grids onto $10 \mu$ drops of the lipid-protein sample. Then the grids were blotted and, after drying, negatively stained with $2.0 \%$ (w/v) uranyl acetate (Sigma). After wicking off the excess stain, the grids were allowed to air-dry and observed under the electron microscope at a $15000-30000 \times$ magnification. Confocal images were acquired using a DMIRE2 Confocal Microscope (Leica TCS SP2) with argon ion lasers and a water immersion objective $63 \times / 1.2 \mathrm{~W}$ (Zeiss). A $488 \mathrm{~nm}$ excitation and 498-550 nm emission wavelengths were used for the NBD-PC fluorescent probe. A $561 \mathrm{~nm}$ excitation and 620-700 nm emission wavelengths were used for the TAMRA fluorescent probe. Finally a $458 \mathrm{~nm}$ excitation and 470-700 nm emission wavelengths were used for the experiments in the presence of ThT.

\section{Conclusions}

Protein interaction with lipid surfaces modifies the physicochemical features of both components, resulting in the alteration of protein structure and in the perturbation of the lipid core in the bilayer. In particular, it is known that basic peptides and proteins are able to interact with negatively charged bilayers thus undergoing substantial structural reorganization as a consequence of the strong local negative potential that affects protein structural stability. ${ }^{61}$ In the case of lysozyme-acidic phospholipid 6 systems, the negative electrostatic potential of the surface markedly weakens the electrostatic interactions contributing to the natively folded protein structure, favouring the structural disassembly of the protein. In addition, the positive charge carried by lysozyme at neutral $\mathrm{pH}$ reduces the local membrane potential and the intrinsic stability of the negatively charged liposomal-protein dispersion. At the same time, the protein insertion into the bilayer affects lipid ordering, resulting in a structural alteration of the membrane.

In this work we investigated these effects by using different concentrations of HEWL incubated with phospholipid bilayers containing different POPC/POPG molar ratios and hence with varying densities of surface negative charge. This approach was meant to discriminate between the effects induced by HEWL on the dispersion stability and those induced by the bilayer with different lipid compositions on HEWL misfolding and aggregation.

Our results indicate that HEWL displays a remarkable fusogenic activity on negative vesicles, above a threshold of negative charge density, resulting in vesicle destabilization and clustering into larger structures, eventually leading to vesicle fusion in about seven days. The CD data showed that, while the above process occurs, HEWL misfolds at the vesicle surface; this effect results in protein structural re-organization into more stable amyloid assemblies that most likely insert into the bilayer, as suggested by phospholipid extraction and membrane fluidity perturbation, and favours vesicle clustering/fusion. The complex effects of membranes on peptide/protein stability strongly depend on the physicochemical properties of the bilayer, including hydrophobicity, surface charge, curvature, density of packing and others. ${ }^{62}$

In brief, negatively charged vesicles attract cationic proteins (HEWL) onto their surfaces. The lipid membrane is an anisotropic environment that forces HEWL polypeptides to align and change their conformation so as to minimize the energies of interactions between polar and non-polar sites of protein and lipids. Concomitantly, the low dielectricity of the membrane also forces lysozyme to establish intramolecular hydrogen bonding by folding into $\alpha$-structure, which leads to fusion between vesicles. After that, a slower process of aggregation and clusterization (oligomerization) leads to amyloid fibril formation. ${ }^{61}$

In our case we only investigated the effects of the surface charge and found that its modulation affects HEWL destabilization and propensity to aggregate. In fact, HEWL displayed a different aggregation propensity in the presence of pure POPG or of $10: 90 \mathrm{POPC} / \mathrm{POPG}$ vesicles, respectively, the latter being more efficient, as shown by the $\mathrm{CD}$ and ThT data. TEM images confirmed the different effect of pure POPG or 10:90 POPC/ POPG vesicles on HEWL aggregation. They also showed pure POPG vesicle clustering and then fusion in the presence of HEWL, generating fibrillar structures upon increasing the lipid : vesicle ratio. The lack of interaction of HEWL with pure POPC vesicles was confirmed by CLSM images; in contrast, the protein exhibited a fusogenic activity inducing aggregation of pure POPG GUVs with lipid extraction, as shown by the imaging of the fluorescently labeled probe at the junction areas between the vesicles, preceding vesicle fusion and disassembly into microvesicles. In particular, CLSM investigation allowed for the first time, to the best of our knowledge, the in situ observation of the HEWL/GUV fusion mechanism and the acquisition of 3D images of large filamentous structures, whose composition could be determined, indicating a protein-lipid co-localization.

Lipid withdrawal has been repeatedly demonstrated in natural and synthetic membranes upon interaction with monomeric proteins or their oligomeric aggregates, and it is indicative of bilayer destabilization preceding membrane permeabilization or vesicle aggregation. ${ }^{63}$

Overall, these considerations recall the behaviour of serum amyloid A (SAA), a family of acute-phase proteins whose plasma levels increase dramatically in chronic inflammatory diseases leading to its aggregation in multiple organs and tissues in AA amyloidosis. ${ }^{64}$ SAA and some variants have been proposed to kill invading bacteria upon aggregating at their plasma membrane, a possible explanation of the role of this acute phase protein. ${ }^{65}$ 
Accordingly a better knowledge of the molecular and physicochemical basis of lysozyme-membrane interaction and their consequences at the molecular level will be useful for possible pharmacological exploitation of this protein as well as for the search of molecules able to protect against lysozyme aggregation.

\section{Acknowledgements}

This work was financially supported by FIRB (Italnanonet), MIUR (PRIN 20087K9A2J), Fondazione Cassa di Risparmio di Firenze and CSGI.

\section{References}

1 M. Stefani, Biochim. Biophys. Acta, Gen. Subj., 2004, 1739, 5-25.

2 L. C. Serpell, Biochim. Biophys. Acta, Gen. Subj., 2000, 1502, 16-30.

3 O. Zachörnig, G. Paasche, C. Thieme, N. Korb and K. Arnold, Colloids Surf., B, 2005, 42, 69-78.

4 C. C. Blake, D. F. Koening, G. A. Mair, A. C. North, D. C. Philips and V. R. Sarma, Nature, 1965, 206, 757-766.

5 N. C. Strynadka and M. N. James, J. Mol. Biol., 1991, 220, 401-424.

6 F. Tanaka, L. S. Forster, P. K. Pal and J. Rupley, J. Biol. Chem., 1975, 250, 6977-6982.

7 M. B. Pepys, P. N. Hawkins, D. R. Booth, D. M. Vigushin, G. A. Tennent and A. K. Soutar, Nature, 1993, 362, 553-557.

8 S. Valleix, S. Drunat, J. B. Philit, D. Adoue, J. C. Piette and D. Droz, Kidney Int., 2002, 61, 907-912.

9 M. Yazaki, S. A. Farrell and M. D. Benson, Kidney Int., 2003, 63, 1652-1657.

10 G. Merlini and V. Bellotti, Clin. Chim. Acta, 2005, 357, 168-172.

11 C. Röcken, K. Becker, M. Fändrich, V. Schroeckh, B. Stix and T. Rath, Hum. Mutat., 2006, 27, 119-120.

12 P. Mudgil, M. Torres and T. Millar, Colloids Surf., B, 2006, 48, $128-$ 137.

13 C. F. Wertz and M. Santore, Langmuir, 2002, 18, 1190-1199.

14 T. Arvinte, K. Hildenbrand, P. Wahl and C. Nicolau, Proc. Natl. Acad. Sci. U. S. A., 1986, 83, 962-966.

15 E. Posse, B. F. De Arcuri and R. D. Morero, Biochim. Biophys. Acta, Gen. Subj., 1994, 1193, 101-106.

16 G. F. Vechetti, B. F. de Arcuri, E. Posse, J. L. Arrondo and R. D. Morrero, Mol. Membr. Biol., 1997, 14, 137-142.

17 G. P. Gorbenko, V. M. Loffe and P. K. Kinnunen, Biophys. J., 2007, 93, 140-153.

18 S. Adams, A. M. Higgins and R. A. L. Jones, Langmuir, 2002, 18, 4854-4861.

19 J. J. Bergers, M. H. Vingerhoeds, L. van Bloois, J. N. Herron, L. H. Janssen, M. J. Fischer and D. J. Crommelin, Biochemistry, 1993, 32, 4641-4649.

20 H. Zhao, E. K. J. Tuominen and P. K. J. Kinnunen, Biochemistry, 2004, 43, 10302-10307.

21 R. Kuboi, T. Mawatari and M. Yoshimoto, J. Biosci. Bioeng., 2000, 90, 12-19.

22 B. A. Vernaglia, J. Huang and E. D. Clark, Biomacromolecules, 2004, 5, 1362-1370.

23 E. Frare, M. F. Mossuto, P. P. de Laureto, M. Dumoulin, C. M. Dobson and A. Fontana, J. Mol. Biol., 2006, 361, 551-561.

24 S. Goda, K. Takano, Y. Yamagata, R. Nagata, H. Akutsu, S. Maki, K. Namba and K. Yutani, Protein Sci., 2000, 9, 369-375.

25 T. Gulik-Krywicki, E. Shechter and V. Luzzati, Nature, 1969, 223, 1116-1121.

26 A. M. Melo, M. Prieto and A. Coutinho, Biochim. Biophys. Acta, Gen. Subj., 2011, 1808, 2559-2568.

27 H. K. Kimelberg and D. Papahadjopoulos, Biochim. Biophys. Acta, Biomembr., 1971, 233, 805-809.

28 E. H. W. Pap, M. C. Houbiers, J. S. Santema, A. van Hoek and A. J. W. G. Visser, Eur. Biophys. J., 1996, 24, 223-231.

29 O. Zschörnig, G. Paasche, C. Thieme, N. Korb and A. Arnold, Colloids Surf., B, 2005, 42, 69-78.
30 M. Dumoulin, D. Canet, A. M. Last, E. Pardon, D. B. Archer, S. Muyldermans, L. Wyns, A. Matagne, C. V. Robinson, C. Redfield and C. M. Dobson, J. Mol. Biol., 2005, 346, 773-788.

31 G. P. Gorbenko and P. K. J. Kinnunen, Chem. Phys. Lipids, 2006, 141, 72-82.

32 R. Jahn and P. I. Hanson, Nature, 1998, 393, 14-15.

33 W. Nickel, T. Weber, J. A. McNew, F. Parlati, T. H. Sollner and J. E. Rothman, Proc. Natl. Acad. Sci. U. S. A., 1999, 96, 12571-12576.

34 F. Parlati, T. Weber, J. A. McNew, B. Westermann, T. H. Sollner and J. E. Rothman, Proc. Natl. Acad. Sci. U. S. A., 1999, 96, 12565-12570.

35 K. Arnold, D. Hoekstra and S. Ohki, Biochim. Biophys. Acta, Gen. Subj., 1992, 1124, 88-94.

36 E. Posse, V. A. Lopez, B. F. de Arcuri, R. N. Farias and R. D. Morero, Biochim. Biophys. Acta, Gen. Subj., 1990, 1024, 390-394.

37 H. Zhao, A. Jutila and T. Nurminen, Biochemistry, 2005, 44, $2857-$ 2863.

38 H. R. Ibrahim, M. Yamada, K. Matzushita, K. Kobayashi and A. Kato, J. Biol. Chem., 1994, 269, 5059-5063.

39 T. Pillot, L. Lins, M. Goethals, B. Vanloo, J. Baert, J. Vandekerckhove, M. Rosseneu and R. Brasseur, J. Mol. Biol., 1997, 274, 381-393.

40 S. Dante, T. Hauss, A. Brandt and N. A. Dencher, J. Mol. Biol., 2008, 376, 393-404.

41 J. Repáková, J. M. Holopainen, M. R. Morrow, M. C. McDonald, P. Capková and I. Vattulainen, Biophys. J., 2005, 88, 3398-3410.

42 W. Witoonsaridsilp, P. Busaba, S. Narong and C. C. M. Goyma, Colloids Surf., B, 2010, 75, 501-509.

43 G. P. Gorbenko, V. M. Ioffe and P. K. J. Kinnunen, Biophys. J., 2007, 93, $140-153$

44 V. M. Trusova, G. P. Gorbenko, I. Akopovac, J. G. Molotkovsky, I. Gryczynskic, J. Borejdoc and Z. Gryczynskic, Colloids Surf., B, 2010, 80, 219-226.

45 N. Chaudhary and R. Nagaraj, Mol. Cell. Biochem., 2009, 328, 209-215.

46 S. V. Chochina, N. A. Avdulov, U. Igbavboa, J. P. Cleary, E. O. O'Hare, and W. G. Wood, J. Lipid Res., 2001, 42, 1292-1297.

47 K. Wong, Y. Qiu, W. Hyun, R. Nixon, J. VanCleff, J. Sanchez-Salazar, S. B. Prusiner and S. DeArmond, J. Neurol., 1996, 47, 741-750.

48 B. L. Kagan, in Protein Misfolding and Conformational Diseases Part A: Protein Aggregation and Conformational Diseases, ed. V. N. Uversky and A. L. Fink, 2006, pp. 223-233.

49 G. Gorbenko and V. Trusova, Biophys. Chem., 2011, 154(2-3), 73-81.

50 G. V. Trusova, Biophys. Chem., 2011, 154(2-3), 73-81.

51 G. P. Gorbenko, V. M. Ioffe, J. G. Molotkovsky and P. K. Kinnunen, Biochim. Biophys. Acta, Gen. Subj., 2008, 1778, 1213-1221.

52 M. Bokvist, F. Lindström, A. Watts and G. Gröbner, J. Mol. Biol., 2004, 335, 1039-1049.

53 M. Groenning, J. Chem. Biol., 2010, 3, 1-18.

54 R. Sabate and S. J. Saupe, Biochem. Biophys. Res. Commun., 2007, 360, 135-138.

55 V. M. Trusova, G. P. Gorbenko, P. Sarkar, R. Luchowski, I. Akopova, L. D. Patsenker, O. Klochko, A. L. Tatarets, Y. O. Kudriavtseva, E. A. Terpetschnig, I. Gryczynki and Z. Gryczynski, J. Phys. Chem. B, 2010, 114, 16773-16782.

56 S. Nappini, T. Al Kayal, D. Berti, B. Norden and P. Baglioni, J. Phys. Chem. Lett., 2011, 2, 713-718.

57 M. I. Angelova and D. S. Dimitrov, Prog. Colloid Polym. Sci., 2007, 76, 59-67.

58 M. I. Angelova, S. Soleau, P. Meleard, J. F. Faucon and P. Bothorel, Trends in Colloids and Interfaces Science, 1992, 89, 127-131.

59 F. Baldelli Bombelli, D. Berti, F. Pini, U. Keiderling and P. Baglioni, J. Phys. Chem. B, 2004, 108, 16427-16434.

60 S. W. Provencher, Comput. Phys. Commun., 1982, 27, 213-227.

61 P. K. J. Kinnunen, Open Biol. J., 2009, 2, 163-175.

62 S. Tamamiro-Kato, M. G. Kosaraju, H. Kato, V. Vincent Raussens, J. M. Ruysschaert and V. Narayanaswami, Biochemistry, 2006, 45, 10947-10956.

63 B. B. Bonev, R. J. C. Gilbert, P. W. Andrew, O. Byron and A. Watts, J. Biol. Chem., 2001, 278, 5714-5719.

64 R. Myllykangas-Luosujarvi, K. Aho, H. Kautiainen and M. Hakala, Rheumatology, 1999, 38, 499-503.

65 Y. Hirakura, I. Carreras, J. D. Sipe and B. L. Kagan, Amyloid, 2002, 9, 13-23. 